Picture an enzyme at work

\section{Guy Riddihough}

OIL and water don't mix. So how does pancreatic lipase, an enzyme that knocks around in the essentially aqueous environment of the small intestine, get to grips with fats and oils and break them down into the glycerol and fatty acids that are readily absorbed by the lining of the gut? Several elements of the answer come together in the paper by $\mathrm{H}$. van Tilbeurgh and colleagues on page 814; this is a sequel to the same group's report of last year (Nature 359, 159-162; 1992), discussed in News and Views in the same issue (page 107).

The catalytic activity of pancreatic lipase, like that of a number of other lipases, is greatly increased when the enzyme comes into contact with a lipid/ water interface - this is the phenomenon known as interfacial activation. van Tilbeurgh et al. have now crystallized and determined the structure of a human pancreatic lipase-colipase complex in the presence of mixed phospholipid/bile salt micelles. The results tell us a great deal about the mechanistic basis of interfacial activation.

The presence of the mixed micelles in the crystallization solution induces a

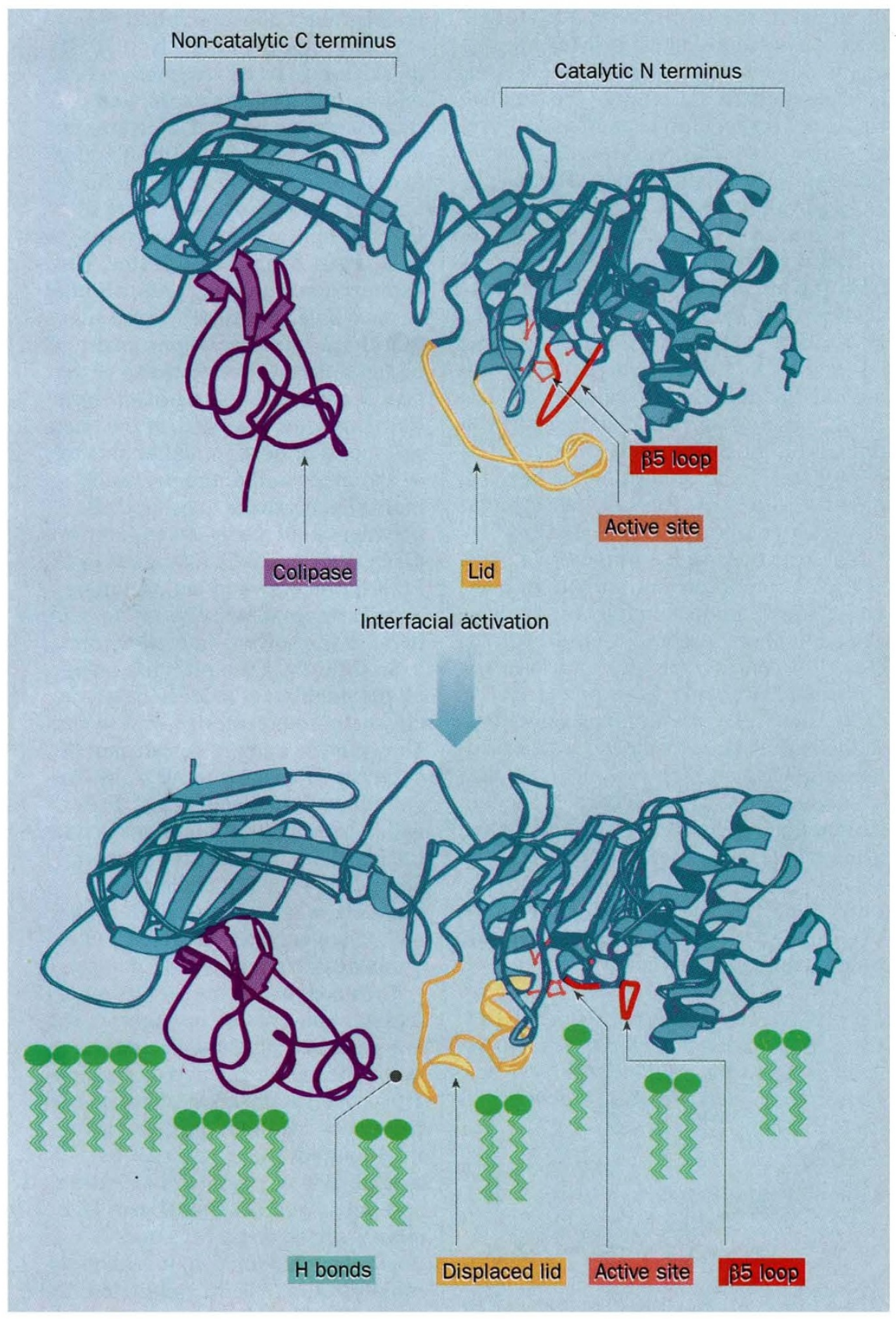

series of conformational rearrangements in the protein complex which, in combination, create a binding site for the lipid substrate. The most dramatic change is seen in the $\alpha$-helical 'lid' (yellow in the figure), which covers the active site of the enzyme and blocks access to the substrate in the 'closed', inactive, complex. In the presence of micelles (green) the lid pops open. This movement exposes the active site (pink), consisting of a catalytic triad like that of the serine proteinases, which lies at the bottom of a hydrophobic canyon in the catalytic amino-terminal domain of the protein. Furthermore, in the structure there is a region of electron density which could be interpreted as a phospholipid molecule in the canyon, with its two acyl chains sitting comfortably in hydrophobic grooves and the scissile carbonyl carbon positioned in front of the active-site serine $\gamma$-oxygen.

A further consequence of the opening of the lid is the restructuring of the $\beta 5$-loop (red in the figure). In the closed complex, this loop makes van der Waals contacts exclusively with the lid. When the lid opens these contacts are lost and the loop folds back onto the core of the protein. This creates an electrophilic region, the 'oxyanion hole', around the active site serine; the oxyanion hole helps stabilize the transition-state intermediate formed during catalysis. The movements of the lid and the $\beta 5$-loop expose hydrophobic residues and bury hydrophilic ones on the active-site face of the complex, further increasing the affinity of the complex for the lipid substrate.

The activity of pancreatic lipase is inhibited by a number of components in the small intestine, among them bile salts, secreted by the gall bladder. The inhibitory effect of these components is overcome by colipase, a small protein also secreted by the exocrine pancreas, which binds to the non-catalytic carboxyterminal domain of the enzyme (purple in the figure).

Colipase has three hydrophobic 'fingers' which point out from the protein complex on the same face as the active site of the lipase. These fingers are thought to enhance binding of the complex to the surface of the micelles, overcoming the inhibitory effect of bile salts. The new structure reveals a second important role for colipase. Once bound, interfacial activation brings the colipase molecule close to the lid and three hydrogen bonds between the fingers and the lid stabilize the conformation of the open complex. This interaction seems to be crucial for the activity of the enzyme.

Guy Riddihough is an assistant editor of Nature. 